\title{
Correction to: Reconnaissance of geotechnical aspects of the 2016 Central Italy earthquakes
}

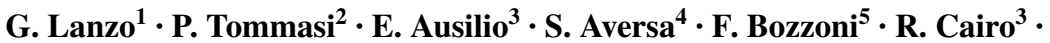 \\ A. d'Onofrio $^{6}$ - M. G. Durante ${ }^{7} \cdot$ S. Foti $^{8} \cdot$ S. Giallini' ${ }^{9}$ M. Mucciacciaro ${ }^{10}$. \\ A. Pagliaroli ${ }^{11}$-S. Sica ${ }^{10}$ - F. Silvestri ${ }^{12}$ - G. Vessia $^{9}$ - P. Zimmaro ${ }^{7}$
}

Published online: 25 April 2018

(C) Springer Science+Business Media B.V., part of Springer Nature 2018

\section{Correction to: Bull Earthquake Eng https://doi.org/10.1007/s10518-018-0350-8}

Because of an error during the editorial process the first name initial of author Ernesto Ausilio was incorrectly given as A. (A. Ausilio) in the initial online publication. It should obviously be E. Ausilio. The original article has been corrected.

The original article can be found online at https://doi.org/10.1007/s10518-018-0350-8.

G. Vessia

g.vessia@unich.it

1 Sapienza Università di Roma, Rome, Italy

2 Consiglio Nazionale delle Ricerche, Rome, Italy

3 Università della Calabria, Rende, Italy

4 Università degli Studi di Napoli Parthenope, Naples, Italy

5 Eucentre, Pavia, Italy

6 Università degli Studi di Napoli Federico II, Naples, Italy

7 University of California Los Angeles (UCLA), Los Angeles, USA

8 Politecnico di Torino, Turin, Italy

9 Università di Chieti-Pescara, Pescara, Italy

10 Università del Sannio, Benevento, Italy

11 Università degli Studi di Chieti-Pescara, Pescara, Italy

12 Università di Napoli Federico II, Naples, Italy 